\title{
Carcinoma della prostata: prostatectomia radicale nei pazienti over 70
}

Massimo Madonia ${ }^{1}$, Paolo Soggia ${ }^{1}$, Gianmarco Garau ${ }^{1}$, Sebastiano Cimino 2 , Giuseppe Morgia ${ }^{2}$, Carlo Corbu ${ }^{1}$

Istituto di Clinica Urologica, Azienda Ospedaliero Universitaria di Sassari

Dipartimento di Urologia, Università degli studi di Catania

\section{Abstract}

Prostate cancer is one of the most common male cancers in industrialized countries and it occurs mainly in men older than 50 years. In light of a rapidly ageing population it seems certain tha increasingly many septuagenarians with significant life-expectancy will present with prostate cancer to urologist. However currently there is a lack of consensus on prostate cancer and its treatment in the cohort of men $\geq 70$ years of age. Although elderly men are often recommended a watchfull waiting option based on lower life expentancy and potentially slow disease progression, recent evidences indicate suggest a potential benefit of radical prostatectomy in selected older patients. The aim of this review is to illustrate the evidences demonstrating the efficacy and safety and of radical prostatectomy in patients over 70. Also, are presented the results of a small Italian study on patients aged $\geq 70$ years with clinically localized prostate cancer undergoing radical prostatectomy. The results show that radical prostatectomy is a safe and effective option in elderly patients, with low incidence of complications and recovery of continence in about two-thirds of patients.

\section{Keywords}

Radical prostatectomy; Prostate cancer; Elderly men 


\section{Introduzione}

Il tumore della prostata è la neoplasia più frequente nella popolazione maschile dei paesi industrializzati [1] e in Italia rappresenta il 20\% di tutti i tumori diagnosticati nei maschi over 50 [2]. Negli ultimi anni si è assistito a un progressivo invecchiamento demografico della popolazione e nei prossimi decenni la popolazione con più di 65 anni sarà sempre più numerosa, passando dal $20,3 \%$ del 2011 al 22,7\% nel 2021, fino al 32,6\% nel 2065 [3]. Per quanto riguarda la popolazione maschile $\geq 65$ anni le previsioni demografiche dell'ISTAT prevedono che passerà dai 5.190 .942 del 2011, ai 6.171.483 del 2021 fino agli 8.769 .090 del 2065. Analogamente è stato osservato un notevole incremento dell'aspettativa di vita e attualmente un uomo di 65 anni ha una speranza di vita di 18,5 anni [3]. Tutti questi cambiamenti demografici portano necessariamente a dover riconsiderare lapproccio terapeutico al carcinoma della prostata nei pazienti più anziani che, a oggi, tende a essere alquanto conservativo.

Obiettivo di questa review è illustrare i dati presenti in letteratura che dimostrano l'efficacia e la sicurezza, nonché l'esigenza clinica, dell'intervento di prostatectomia radicale anche nei pazienti over 70 . Inoltre, vengono presentati i dati di una casistica italiana che confermano che la prostatectomia in questa tipologia di pazienti, con poche morbilità associate, rappresenta unalternativa sicura, curativa $\mathrm{e}$ con poche complicanze legate all'intervento, nella maggior parte dei casi.

\section{Il carcinoma della prostata}

\section{Incidenza e mortalità}

A partire dai primi anni '90 la diffusione di nuove procedure diagnostiche, quali il test del PSA e la biopsia prostatica ecoguidata, hanno portato a un netto aumento del numero di diagnosi di carcinoma prostatico [1] e questa tendenza sembra destinata ad aumentare passando dai circa 36 mila nuovi casi che erano attesi nel 2012 a circa 44 mila nel 2020 e a quasi 52 mila nel 2030 [2].

Sebbene lincidenza del tumore alla prostata sia tra le più alte rispetto ad altri tipi di tumore, la mortalità, stimata attorno ai 17-18 decessi ogni 100.000 abitanti/anni, è solo al terzo posto e riguarda principalmente pazienti over 70 . Tuttavia, a causa delletà dei pazienti e delle comorbidità generalmente presenti in questa popolazione, non è semplice separare i decessi per tumore dai decessi nei pazienti con tumore [2]. Non considerando la mortalità per altre cause la sopravvivenza a 5 anni dalla diagnosi è dell' $88 \%$, con una percentuale di sopravvivenza che è maggiore fino ai 74 anni di età per diminuire a $84 \%$ nella fascia d'età $75-84$ e al 51\% tra gli 85-99enni [4].

I tassi di incidenza e sopravvivenza del tumore alla prostata variano notevolmente tra i diversi paesi europei a causa, probabilmente, della diversa diffusione e del diverso utilizzo del dosaggio del PSA come strumento di screening che permette l'identificazione di tumori asintomatici probabilmente meno aggressivi dei tumori diagnosticati clinicamente [5].

\section{Sintomatologia e diagnosi}

Nella fase iniziale il carcinoma della prostata è asintomatico e viene diagnosticato in seguito a esplorazione rettale o dosaggio del PSA eseguiti nel corso di controlli clinici. In seguito a crescita della massa tumorale compaiono i primi sintomi che sono simili a quelli che si riscontrano nell'ipertrofia prostatica benigna e che comprendono difficoltà a urinare, dolore alla minzione, frequente necessità di urinare, presenza di sangue nelle urine, indebolimento del getto [6,7]. 
Nei pazienti che presentano sintomi riferibili a patologia prostatica la prima indagine diagnostica da effettuare è l'esplorazione rettale, seguita da esami via via più specifici, quali il dosaggio del PSA, le tecniche per immagini (ecografia transrettale, TC e MRI) e l'agobiopsia prostatica [6].

\section{Classificazione}

Una volta instaurata la diagnosi è necessario stabilire il grado e lo stadio del tumore in modo da valutare la classe di rischio del paziente e definire lapproccio terapeutico più adatto. In particolare, il grado istologico del tumore alla prostata viene valutato mediante il Gleason score che prende in considerazione il grado di differenziazione ghiandolare e il tipo di infiltrazione. Il punteggio del Gleason score va da 2 a 10, più basso è il punteggio, minore è il grado di tumore [8]. Lo stadio del tumore viene attribuito mediante la classificazione TNM che prende in considerazione l'estensione del tumore primario, il coinvolgimento linfonodale e le metastasi a distanza [9]. Sulla base del Gleason score, dellestensione tumorale e dei livelli di PSA viene identificata la classe di rischio, come riportato in Tabella I.

\section{Terapia chirurgica}

La prostatectomia radicale consiste nella rimozione chirurgica totale della prostata e delle vescicole seminali e dei linfonodi pelvici se il rischio di linfonodi positivi è superiore al 5\% [6]. Può essere eseguita a cielo aperto, laporoscopica o robot assistita.

La prostatectomia radicale consente di ottenere il miglior controllo di malattia a lungo termine in caso di carcinoma alla prostata clinicamente localizzato [10], tuttavia il rischio di complicanze post-operatorie, quali l'incontinenza o l'impotenza, spesso fungono da deterrente al trattamento. Negli anni sono stati messi a punto numerosi nomogrammi in grado di predire loutcome dell'intervento di prostatectomia radicale, sulla base delle caratteristiche del singolo paziente, in modo da scongiurare al massimo il rischio di complicanze [10]. In particolare, secondo uno studio prospettico tedesco, nel quale sono stati presi in esame i dati di 236 pazienti che erano stati sottoposti a prostatectomia radicale retropubica open, i fattori in grado di influenzare positivamente loutcome in termini di qualità di vita, potenza sessuale e continenza a 1 anno dall'intervento, sono risultati essere PSA preoperatorio $<20 \mathrm{ng} / \mathrm{ml}$, tecnica nerve sparing, assenza di complicanze intra- o post-operatorie, assenza di trattamento adiuvante e partecipazione a un programma di riabilitazione postoperatoria [10]. Nello studio inoltre non è stata riscontrata unassociazione tra il recupero della continenza e l'età del paziente [10].

Secondo le linee guida i candidati ideali all'intervento di prostatectomia radicale sono $i$ pazienti con neoplasie a rischio intermedio (cT2b-cT2c o Gleason score $=7$ o PSA $=10-20$ $\mathrm{ng} / \mathrm{ml}$ ) e aspettativa di vita superiore ai $10 \mathrm{anni}$, ma, come riportato in Tabella II non si esclude il trattamento nei pazienti in altri stadi, se le caratteristiche cliniche e anatomo-patologiche della neoplasia sono indicative di outcome favorevole [8].

\begin{tabular}{|lccccc|}
\hline $\begin{array}{c}\text { Classe } \\
\text { di rischio }\end{array}$ & Stadio & & $\begin{array}{c}\text { Gleason } \\
\text { score }\end{array}$ & $\begin{array}{c}\text { PSA } \\
(\mathrm{ng} / \mathrm{ml})\end{array}$ \\
Basso & cT1-cT2a & e & $\leq 6$ & e & $<10$ \\
Intermedio & cT2b-cT2c & e/o & $=7$ & e/o & $10-20$ \\
Alto & cT3a-cT3b-cT4 & e/o & $>7$ & e/o & $>20$ \\
\hline
\end{tabular}

Tabella I. Classi di rischio nel tumore alla prostata [8]

\begin{tabular}{|ll|}
\hline Indicata & cT1 $\mathrm{a}-\mathrm{T} 2 \mathrm{~b}+$ Gleason $=2-7+$ \\
& PSA $\leq 20 \mathrm{ng} / \mathrm{ml}$ e aspettativa di vita \\
Opzionale & cT10 anni \\
& $\begin{array}{l}\text { CT1a, aspettativa di vita }>15 \text { anni o } \\
\text { Gleason }=7\end{array}$ \\
& cT3a o Gleason $=8-10$ o PSA $>20 \mathrm{ng} / \mathrm{ml}$ \\
& $\begin{array}{l}\text { cT3b-cT4 N0 o per ogni TN1 (nell'ottica } \\
\text { di un trattamento multimodale) }\end{array}$ \\
Raccomandazioni & $\begin{array}{l}\text { Chirurgia nerve-sparing in pz con cT1c, } \\
\text { Gleason }<7 \text { e PSA }<10 ~ n g / m l\end{array}$ \\
& $\begin{array}{l}\text { Nerve-sparing unilaterale opzionale in } \\
\text { pz cT2a-cT3a }\end{array}$ \\
\hline
\end{tabular}

Tabella II. Indicazioni alla prostatectomia radicale [8] 
In generale la sopravvivenza dopo un intervento di prostatectomia radicale è molto elevato e le cause di morte negli anni successivi sono dovuti principalmente a cause non correlate al tumore [11]. Nello studio di Eifler del 2012, lanalisi dei dati di più di 18 mila pazienti sottoposti a prostatectomia radicale tra il 1975 e il 2009 ha evidenziato un tasso di morte del 7,8\% (1.419 pazienti) a un follow up mediano di 7,4 anni, di questi pazienti solo 379 sono morti a causa del tumore alla prostata [11]. Il confronto della mortalità in questa coorte di pazienti e la mortalità generale della popolazione americana ha evidenziato un tasso leggermente inferiore nei pazienti sottoposti a prostatectomia radicale (Tasso di mortalità standardizzato $=0,47 ; \mathrm{p}<0,05)$ a dimostrazione del beneficio dell'intervento chirurgico in alcune tipologie di pazienti [11]. Lo studio dello Scandinavian Prostate Cancer Group ha dimostrato che negli uomini con carcinoma alla prostata localizzato che sono stati sottoposti a prostatectomia radicale, la mortalità cancro-correlata è significativamente minore, rispetto alla stessa tipologia di pazienti che non hanno ricevuto alcuna terapia specifica ma solo una vigile attesa [12]. In particolare l'incidenza cumulativa di morte per cancro alla prostata dopo 15 anni di follow up è risultata del 14,6\% nei pazienti sottoposti a intervento chirurgico e del 20,7\% nel gruppo in vigile attesa $(R R=0,62, p=0,01)$ [12].

\section{Prostatectomia radicale nei pazienti over 70}

A causa del lungo decorso della malattia, i pazienti che maggiormente beneficiano del trattamento radicale in caso di patologia localizzata sono quelli che hanno la più alta aspettativa di vita. Pertanto nella scelta del trattamento da intraprendere è necessario prendere in considerazione non solo lo stadio clinico, il PSA e il risultato della biopsia, ma anche l'aspettativa di vita del paziente [11], generalmente se è $\geq 10$ anni si prende in considerazione la possibilità di un intervento curativo [13].

Negli ultimi anni si è assistito a un notevole incremento dell'aspettativa di vita e ciò porta necessariamente a dover riconsiderare l'approccio terapeutico del carcinoma alla prostata nei pazienti più anziani, che a oggi tende a essere alquanto conservativo [14]. A causa della storia naturale relativamente lunga della patologia e dellopinione diffusa che negli over 70 la patologia sia biologicamente meno ag-

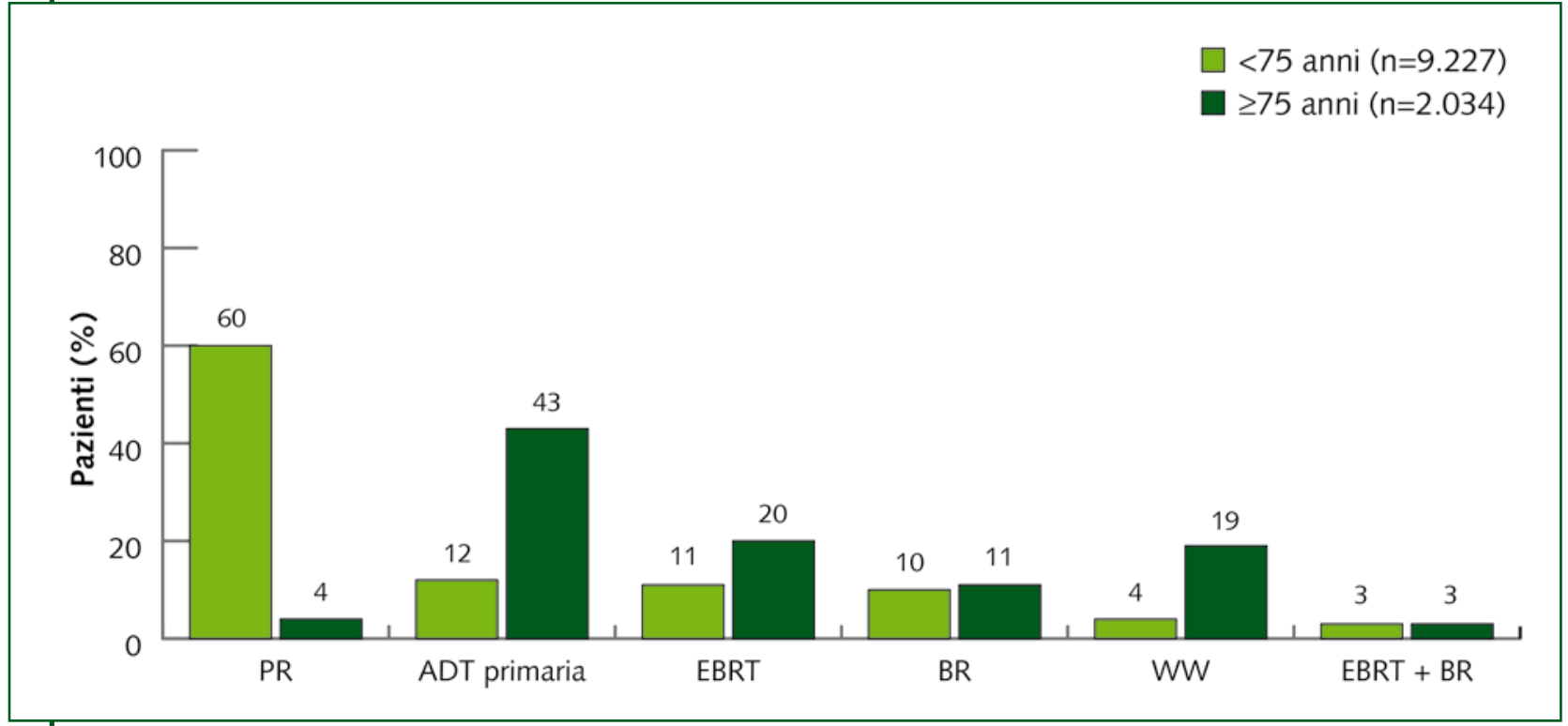

Figura 1. Trattamento primario per categoria ed età nell'analisi di Konety, 2008. Modificato da [15] $\mathrm{ADT}$ = terapia di deprivazione androgenica; $\mathrm{BR}=$ brachiterapia; $\mathrm{EBRT}$ = radioterapia a fasci esterni; $\mathrm{PR}=$ prostatectomia radicale; $\mathrm{WW}=$ watchfull waiting 
gressiva, spesso in questi pazienti non viene infatti intrapreso un trattamento primario [14,15]. Alcuni studi hanno evidenziato che più del $15 \%$ dei pazienti con tumore alla prostata e più di 75 anni sono sottotrattati [15] e che pazienti $<60$ anni con patologia clinicamente localizzata hanno una probabilità 25 volte maggiore di ricevere una prostatectomia radicale, rispetto a pazienti $\geq 70$ anni [16].

Lo studio di Konety del 2008, sulla base dei dati raccolti nel database CaPSURE ha esaminato gli schemi di trattamento primario e secondario nei pazienti $\geq 75$ anni confrontandoli con quelli di pazienti più giovani [15]. Sono stati analizzati i dati di 11.261 pazienti, di cui circa il $18 \%$ aveva più di 75 anni. Le caratteristiche alla baseline hanno rivelato che nei pazienti più anziani erano stati classificati più tumori alla prostata ad alto rischio, rispetto ai pazienti $<75$ anni $(43 \%$ vs 25\%) in quanto PSA, Gleason score e stadio tumorale erano più elevati. Ciò nonostante le tipologie di trattamento a cui è stata sottoposta la coorte variano notevolmente nei due range di età, come illustrato in Figura 1. Lanalisi di regressione dei dati ha inoltre evidenziato che, indipendentemente dalla classe di rischio e dalle comorbilità, nei pazienti con più di 75 anni il trattamento primario più utilizzato era il watchfull waiting [15].

Lo studio di D’Amico del 2003 ha evidenziato che nei pazienti con età $>73$ anni l'età rappresenta un fattore di rischio indipendente di mortalità per tumore alla prostata localizzata nei pazienti che sono stati sottoposti a radioterapia [17]. Infine uno studio canadese ha evidenziato che i pazienti con più di 70 anni e un Gleason score di 8-10 hanno il maggior rischio di sottotrattamento e la più elevata perdita di aspettativa di vita aggiustata per qualità di vita e conclude ipotizzando un potenziale beneficio della prostatectomia radicale in questa categoria di pazienti [18].

Le caratteristiche patologiche del tumore alla prostata nei pazienti con più di 75 anni sono state esaminate anche nello studio di Delongchamps del 2009 che ha valutato 211 prostate derivanti da reperti autoptici di uomini con più di 70 anni $(35 \%)$ o più giovani.

L'incidenza di tumore alla prostata è risultata più elevata nei pazienti più anziani $(45 \% v s 19 \% ; \mathrm{p}<0,001)$, inoltre in questa tipologia di pazienti il carcinoma è risultato essere più grande, in stadio più avanzato e meno differenziato [19]. Lo studio di Brassel del 2011 che aveva come obiettivo la definizione della caratteristiche clinico patologiche nei pazienti con più di 70 anni con tumore alla prostata [20], ha preso in esame i dati di più di 12 mila pazienti a cui era stato diagnosticato tumore alla prostata negli anni compresi tra 1989 e il 2009. Di questi circa il 30\% aveva 70 o più anni e l'analisi dei loro dati, confrontati con quelli di pazienti più giovani, ha mostrato caratteristiche più aggressive in termini di stadio clinico, stadiazione alla biopsia e velocità di PSA $(\mathrm{p}<0,0001)$. Inoltre negli 899 pazienti over 70 che sono stati sottoposti a prostatectomia radicale, è stato evidenziato uno stadio patologico più elevato, un peggiore stato dei margini chirurgici e una stadiazione peggiore rispetto ai pazienti più giovani $(\mathrm{p}<0,0001)$ [20]. Sulla base delle caratteristiche del tumore alla prostata nei pazienti over 70 sopra illustrate, e dellaumento nell'aspettativa di vita in questa categoria di pazienti, appare appropriato considerare il trattamento chirurgico anche nei pazienti più anziani, ovviamente dopo accurata selezione del singolo paziente e delle caratteristiche individuali. Numerosi studi hanno infatti evidenziato come un intervento di prostatectomia radicale risulti efficace e sicuro anche nei pazienti meno giovani. Un esempio è lo studio di Kunz del 2012 che ha investigato l'influenza di un'età $>70$ anni sulle caratteristiche del carcinoma alla prostata e sugli outcome oncologici e funzionali nei pazienti che sono stati sottoposti a prostatectomia radicale retropubica [14]. Lo studio ha preso in esame i dati di 1.636 pazienti e ha confrontato gli outcome e le caratteristiche oncologiche e funzionali tra coloro che avevano 70 o più anni (411 pazienti) e quelli più giovani (1.225 pazienti). Lanalisi dei dati ha evidenziato che i tumori nei pazienti $\geq 70 \mathrm{anni}$ erano significativamente più palpabili e non-organo confinati ( $\mathrm{p}=0,030 \mathrm{e} \mathrm{p}=0,026$, rispettivamente). Lanalisi multivariata ha inoltre mostrato che l'età più avanzata non rappresenta una predittore indipendente di sopravvivenza globale $(\mathrm{p}=0,102)$ e di sopravvivenza cancro-specifica $(\mathrm{p}=0,195)$, come invece sono risultati essere le caratteristiche del tumore (metastasi ai linfonodi, Gleason score $=8-1$ ). La continenza a due anni dall'operazione è risultata comparabile nei due gruppi di pazienti $(\mathrm{p}=0,984)$. I risultati degli autori sono che in considerazione della presenza di patologia più biologicamente ag- 
gressiva nei pazienti $\geq 70$ anni non appare giustificabile escludere questa categoria di pazienti dalla prostatectomia radicale, anche alla luce delle eccellenti outcome postoperatori [14].

Lo studio di Richstone del 2008 ha cercato di determinare l'effetto dell'età sulle caratteristiche clinico patologiche e la sopravvivenza dopo prostatectomia radicale nei pazienti con $70 \mathrm{o}$ più anni. Lo studio ha preso in esame i dati di 4.035 pazienti che erano stati sottoposti a intervento radicale e ha confrontato gli outcome dei pazienti $\geq 70$ anni $(6,4 \%)$ con quelli dei pazienti più giovani [13]. Lanalisi dei dati ha evidenziato che i pazienti $\geq 70$ anni presentavano tumori con più elevato a stadio clinico $(p=0,001)$ e Gleason score $(\mathrm{p}=0,01)$ e una minore frequenza di patologia organo-confinata rispetto agli uomini con meno di 70 anni $(58,1 \%$ vs $69,9 \%$; $\mathrm{p}=0,001)$. È stato inoltre osservato upgrading

Caratteristiche preoperatorie

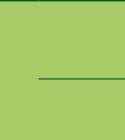

Pazienti
PSA ( $\mathrm{ng} / \mathrm{ml})$

$\leq 4$

$4<$ PSA $\leq 10$

$10<$ PSA $\leq 20$

$>20$

n.

13

14,77

53

60,23

21,59

3,41

Stadio clinico

T1b

T1C

$\mathrm{T} 2 \mathrm{a}$

$\mathrm{T} 2 \mathrm{~b}$

T2C

T3

NS

Gleason Score

Da 2 a 6

$\leq 7$

56

63,63

23

26,13

Da 8 a 10

7

7,95

NS

2

2,27

\section{Classe di rischio}

Basso

24

27,27

Intermedio

69,32

Alto

61

3,41

Tipo intervento

RRP

LERP

70

78,65

19

21,34

Caratteristiche intervento

LAD

Nerve Sparing

43

48,31

3,37 (da Gleason score alla biopsia $=2-6$ a Gleason score patologico $\geq 7$ ) nel $45 \%$ dei pazienti $\geq 70$ anni e nel $35,2 \%$ dei pazienti $(\mathrm{p}=0,01)$ e upgrading (da cT1-T2 a pT3-T4) nel 40,2\% e $29,3 \%$ dei pazienti, rispettivamente $(\mathrm{p}=0,001)$. Non sono state invece osservate differenze tra i due gruppi di pazienti per quanto riguarda la sopravvivenza cancro-specifica (96\% a 10 anni per entrambi; $p=0,003)$ e la probabilità assenza di progressione biochimica ( $74 \%$ e $75 \%$ a 10 anni, rispettivamente; $\mathrm{p}=0,13$ ) [13].

Lo studio di Alibahi del 2003 ha riportato leffetto della prostatectomia radicale e della radioterapia a fasci esterni sullaspettativa di vita e l'aspettativa di vita ponderata per la qualità nei pazienti $\geq 75$ anni [21]. I risultati hanno mostrato che nei pazienti con Gleason score 5-7 la terapia chirurgica e la radioterapia incrementano l'aspettativa di vita, pura e ponderata per la qualità di vita, fino ai 75 anni di età, mentre nel caso di tumori poco differenziati (Gleason score 8-10) il beneficio è chiaro fino agli $80 \mathrm{anni}$ [21] a riprova della teoria che l'età non deve rappresentare una barriera ai trattamenti curativi. Questi risultati indicano pertanto che, in seguito ad attenta selezione dei pazienti, la prostatectomia radicale è un opzione terapeutica da tenere in considerazione anche nei pazienti più anziani.

\section{La casistica italiana}

I benefici di un intervento di prostatectomia radicale in pazienti over 70 con tumore prostatico organo confinato sono stati evidenziati anche in una casistica italiana i cui risultati vengono qui illustrati per la prima volta.

Tabella III. Caratteristiche pre-operatorie e operatorie dei pazienti

$\mathrm{LAD}=$ linfoadenectomia; LERP = prostatectomia

radicale laparoscopica extraperitoneale;

$\mathrm{RRP}=$ prostatectomia radicale retropubica
La casistica include 88 pazienti di età compresa tra i 70 e i 78 anni (età media 72,4 anni) che sono stati sottoposti a prostatectomia radicale tra feb- 
braio 2003 e ottobre 2011 presso la Clinica Urologica di Sassari. Per ogni paziente sono state raccolte le caratteristiche istologiche del tumore alla biopsia (stadio clinico) e sul pezzo anatomico in toto (stadio patologico), il valore di PSA pre- e post-operatorio, eventuali trattamenti ormonali e/o radioterapici postintervento e le complicanze a breve e a lungo termine. Il follow up mediano è stato di 5 anni (range: 9-1). Lanalisi dei dati alla baseline ha evidenziato una prevalenza di tumori organo confinati $(95,4 \%)$ con un Gleason score che nel $90 \%$ dei casi era $\leq 7$ e livelli di PSA $\leq 10 \mathrm{ng} / \mathrm{ml}$ nel $75 \%$ dei pazienti. Sulla base delle caratteristiche dei pazienti alla diagnosi (PSA, stadio clinico e Gleason score) è emersa una prevalenza di tumori a rischio basso $(27,27)$ e intermedio $(69,32)$. Tutti i pazienti sono stati sottoposti a prostatectomia radicale open (78,65\%) o video-laparoscopica (21,34\%); il 48,31\% dei pazienti ha subito linfoadenectomia e solo nel 3,37\% è stato possibile risparmiare i fasci vascolo-nervosi (nerve sparing). La scelta di sottoporre i pazienti a prostatectomia radicale è stata presa sulla base delle caratteristiche antropometriche, sulle morbilità associate, sul performance status di ogni singolo paziente, oltre che sulla base dei dati relativi alla stadiazione qui sotto riportati. Le caratteristiche preoperatorie e operatorie dei pazienti sono riassunte in Tabella III.

L'analisi dei dati dei pazienti dopo l'intervento di prostatectomia radicale ha evidenziato un calo del PSA a valori indosabili nella maggior parte dei casi, è stato osservato PSA $<0,2 \mathrm{ng} / \mathrm{ml}$ in 8 casi $(9,09 \%)$ di cui solo $3(3,43 \%)$ erano $>0,4$ $\mathrm{ng} / \mathrm{ml}$. È stato osservato upgrading da Gleason score 2-6 alla biopsia a $\geq 7$ dopo prostatectomia nel $34,09 \%$ della coorte, coerentemente con le percentuali $30-43 \%$ indicate in letteratura [22], e analogamente anche la percentuale di pazienti che sono passati da malattia clinicamente localizzata a stadio patologico extraprostatico $(32,95 \%)$ è coerente con i dati di letteratura che riportano un range di $24-60 \%$ [22].

Per quanto riguarda la continenza è stata ripresa nel $64,77 \%$ dei casi e nei 31 pazienti (35,22\%) incontinenti $6(19,35 \%)$ presentano incontinenza da stress. Le complicanze post-intervento riscontrate sono state molto poche $(4,55 \%) \mathrm{e}$ comprendevano 1 embolia polmonare, 2 deiescenze delle anastomosi e 1 voluminoso linfocele che ha richiesto puntura percutanea. Infine, 4 pazienti $(4,55 \%)$ hanno ricevuto terapia ormonale con analoghi e $2(2,27)$ radioterapia post-intervento; uno solo $(1,14 \%)$ è stato sottoposto a terapia con bicalutamide.

In Tabella IV sono riportate le caratteristiche e le complicanze post-intervento.

Alla luce dei risultati dellanalisi appare evidente che, in questa casistica di pazienti over 70 con poche morbilità e tumore alla prostata organo

\begin{tabular}{|c|c|c|}
\hline \multirow{2}{*}{$\begin{array}{l}\text { Caratteristiche post- } \\
\text { operatorie }\end{array}$} & \multicolumn{2}{|c|}{ Pazienti } \\
\hline & n. & $\%$ \\
\hline \multicolumn{3}{|l|}{ PSA } \\
\hline$>0,2 \mathrm{ng} / \mathrm{ml}$ & 9 & 10 \\
\hline$>0,4 \mathrm{ng} / \mathrm{ml}$ & 3 & 3,33 \\
\hline \multicolumn{3}{|l|}{ Stadio patologico } \\
\hline $\mathrm{T} 2 \mathrm{a}$ & 10 & 11,36 \\
\hline $\mathrm{T} 2 \mathrm{~b}$ & 27 & 30,68 \\
\hline $\mathrm{T} 2 \mathrm{c}$ & 21 & 23,86 \\
\hline Т3а & 2 & 25 \\
\hline T3b & 6 & 6,81 \\
\hline T3c & 2 & 2,27 \\
\hline NS & 0 & 0,00 \\
\hline Da cT2 a pT3-pT4 & 29 & 32,95 \\
\hline \multicolumn{3}{|l|}{ Gleason Score post } \\
\hline Da 2 a 6 & 32 & 36,36 \\
\hline$\leq 7$ & 42 & 47,72 \\
\hline $\mathrm{Da} 8$ a 10 & 11 & 12,5 \\
\hline NS & 3 & 3,41 \\
\hline Da $2-6$ pre a $\geq 7$ post & 30 & 34,09 \\
\hline \multicolumn{3}{|c|}{ Metastasi ai linfonodi regionali } \\
\hline$N X$ & 26 & 29,54 \\
\hline NO & 59 & 67,04 \\
\hline N1 & 3 & 3,41 \\
\hline \multicolumn{3}{|l|}{ Continenza post } \\
\hline Continente & 57 & 64,77 \\
\hline Incontinente (da stress) & $31(6)$ & $35,22(19,35)$ \\
\hline Complicanze & 4 & 4,55 \\
\hline Radioterapia & 2 & 2,27 \\
\hline In terapia con analoghi & 4 & 4,55 \\
\hline In terapia con bicalutamide & 1 & 1,14 \\
\hline
\end{tabular}

Tabella IV. Caratteristiche post-operatorie 
confinato, la prostatectomia radicale rappresenta un intervento sicuro ed efficace nella maggior parte dei casi, con un'incidenza di complicanze estremamente bassa e ripresa della continenza in circa i due terzi dei pazienti.

\section{Conclusioni}

Il tumore alla prostata è la neoplasia più frequente nella popolazione maschile dei paesi industrializzati [1] e in Italia rappresenta il 20\% di tutti i tumori diagnosticati nei maschi over 50. Il progressivo invecchiamento della popolazione e l'aumento dellaspettativa di vita a cui si assiste negli ultimi anni ha portato i clinici a dover riconsiderare l'approccio terapeutico nei pazienti più anziani con carcinoma alla prostata.

A causa della storia naturale della patologia e dellopinione diffusa che negli over 70 la patologia sia biologicamente meno aggressiva, spesso in questi pazienti non viene infatti intrapreso un trattamento primario. I dati presentati in questa review sfatano un po' queste credenze e a oggi appare chiaro che $\mathrm{i}$ pazienti $>70$ anni, non solo spesso presentano patologie più aggressive rispetto ai soggetti più giovani, $\mathrm{ma}$ anche che possono beneficiare di un intervento curativo quale la prostatectomia radicale. Un'ulteriore conferma arriva da una casistica italiana di pazienti over 70 che sono stati sottoposti a prostatectomia radicale. Anche in questo caso infatti i benefici a lungo termine superano i possibili

Implicazioni per future ricerche

Valutazione della necessità di sottoporre a prostatectomia radicale i pazienti over 80 in buona salute. rischi e le complicanze perioperatorie e a lungo termine, avvalorando l'ipotesi che un intervento curativo primario può portare a eccellenti outcome post-operatori e a un aumento dell'aspettativa e della qualità di vita anche nei pazienti più anziani.

\section{La review in breve}

\begin{tabular}{|c|c|}
\hline Quesito clinico & $\begin{array}{l}\text { Valutazione dell'efficacia e sicurezza dell'intervento di prostatectomia radicale nei pazienti over } 70 \\
\text { con tumore prostatico organo confinato }\end{array}$ \\
\hline Tipologia di revisione & Narrativa \\
\hline $\begin{array}{l}\text { Ricerca della } \\
\text { letteratura }\end{array}$ & $\begin{array}{l}\text { Ricerca su PubMed degli articoli in lingua inglese utilizzando le seguenti parole chiave: radical } \\
\text { prostatectomy, prostate cancer, patients aged } \geq 70 \text {, prostate carcinoma, prostate cancer in elderly } \\
\text { men. Si è fatto inoltre riferimento alle revisioni pubblicate in letterature integrate dalle competenze } \\
\text { ed esperienze degli Autori }\end{array}$ \\
\hline Conclusioni & $\begin{array}{l}\text { Alla luce dei dati la prostatectomia radicale rappresenta un'alternativa terapeutica valida, sicura ed } \\
\text { efficace anche nei pazienti over } 70 \text { con poche morbilità e malattia organo confinata }\end{array}$ \\
\hline Aree grigie & $\begin{array}{l}\text { Mancanza di studi indirizzati alla valutazione delle morbidità e della qualità di vita dopo } \\
\text { prostatectomia radicale nei pazienti }>70 \text { anni }\end{array}$ \\
\hline
\end{tabular}

\section{Bibliografia}

1. Inghelmann R, Grande E, Francisci S, et al. Regional estimates of prostate cancer burden in Italy. Tumori 2007; 93: 380-6; http://dx.doi.org/10.1700/294.3481 
2. AIOM-AIRTUM. I numeri del cancro in Italia, 2012. Brescia: Intermedia, 2012

3. I.Stat. Previsioni demografiche - Anni 2011-2065: Indicatori demografici. Disponibile su: http:// dati.istat.it

4. AIRTUM Working Group. I tumori in Italia - Rapporto 2011. La sopravvivenza dei pazienti oncologici in Italia. Epidemiol Prev 2011; 35 (suppl 3): 1-200

5. Gatta G, Zigon G, Buemi A, et al. Prostate cancer treatment in Europe at the end of 1990s. Acta Oncol 2009; 48: 867-73; http://dx.doi.org/10.1080/02841860902719174

6. Linee guida AIOM. Carcinoma della prostata. 2012

7. Consiglio Nazionale delle Ricerche. Ministero della'Istruzione dell'Università e della Ricerca. Basi scientifiche per linee guida - Prostata. Disponibile su www.progettooncologia.cnr.it

8. Heidenreich A, Bastian PJ, Bellmunt J, et al. Guidelines on Prostate Cancer. European Association of Urology, 2012

9. Sobin LH, Gospodariwicz M, Wittekind C (eds). TNM classification of malignant tumors. UICC International Union Against Cancer. 7th edn. Wiley-Blackwell, 2009; pp. 243-248

10. Treiyer A, Anheuser P, Bütow Z, et al. A single center prospective study: prediction of postoperative general quality of life, potency and continence after radical retropubic prostatectomy. J Urol 2011; 185: 1681-5; http://dx.doi.org/10.1016/j.juro.2010.12.052

11. Eifler JB, Humphreys EB, Agro M, et al. Causes of death after radical prostatectomy at a large tertiary center. J Urol 2012; 188: 798-801; http://dx.doi.org/10.1016/j.juro.2012.04.109

12. Bill-Axelson A, Holmberg L, Ruutu M, et al.; SPCG-4 Investigators. Radical prostatectomy versus watchful waiting in early prostate cancer. N Engl J Med 2011; 364: 1708-17; http://dx.doi. org/10.1056/NEJMoa1011967

13. Richstone L, Bianco FJ, Shah $\mathrm{HH}$, et al. Radical prostatectomy in men aged >or=70 years: effect of age on upgrading, upstaging, and the accuracy of a preoperative nomogram. BJU Int 2008; 101 : 541-6; http://dx.doi.org/10.1111/j.1464-410X.2007.07410.x

14. Kunz I, Musch M, Roggenbuck U, et al. Tumour characteristics, oncological and functional outcomes in patients aged $\geq 70$ years undergoing radical prostatectomy. BJU Int 2013; 111: E24-9; http:// dx.doi.org/ 10.1111/j.1464-410X.2012.11368.x

15. Konety BR, Cowan JE, Carroll PR; CaPSURE Investigators. Patterns of primary and secondary therapy for prostate cancer in elderly men: analysis of data from CaPSURE. J Urol 2008; 179: $1797-$ 803; http://dx.doi.org/10.1016/j.juro.2008.01.044

16. Yan Y, Carvalhal GF, Catalona WJ, et al: Primary treatment choices for men with clinically localized prostate carcinoma detected by screening. Cancer 2000; 88: 1122-30

17. D’Amico AV, Cote K, Loffredo M, et al. Advanced age at diagnosis is an independent predictor of time to death from prostate carcinoma for patients undergoing external beam radiation therapy for clinically localized prostate carcinoma. Cancer 2003; 97: 56-62; http://dx.doi.org/10.1002/cncr.11053

18. Schwartz KL, Alibhai SM, Tomlinson G, et al. Continued undertreatment of older men with localized prostate cancer. Urology 2003; 62: 860-5

19. Delongchamps NB, Wang CY, Chandan V, et al. Pathological characteristics of prostate cancer in elderly men. J Urol 2009; 182: 927-30; http://dx.doi.org/10.1016/j.juro.2009.05.018

20. Brassell SA, Rice KR, Parker PM, et al. Prostate cancer in men 70 years old or older, indolent or aggressive: clinicopathological analysis and outcomes. J Urol 2011; 185: 132-7; http://dx.doi. org/10.1016/j.juro.2010.09.014

21. Alibhai SM, Naglie G, Nam R, et al. Do older men benefit from curative therapy of localized prostate cancer? J Clin Oncol 2003; 21: 3318-27

22. Grossfeld GD, Chang JJ, Broering JM, et al. Under staging and under grading in a contemporary series of patients undergoing radical prostatectomy: results from the Cancer of the Prostate Strategic Urologic Research Endeavor database. J Urol 2001; 165: 851-6 